\title{
Effects of High Dose Fibrinogen on in vitro Haemodilution with Different Therapeutic Fluids
}

\section{Eric Lidgard ${ }^{1}$, Attila Frigyesi ${ }^{2}$ and Ulf Schött ${ }^{3 *}$}

${ }^{1}$ Medical Graduate, Lund University, Skane University Hospital, Department of Anaesthesiology and Intensive Care, S-22185, Lund, Sweden

${ }^{2}$ Lund University, Skane University Hospital, Department of Anaesthesiology and Intensive Care, S-22185, Lund, Sweden

${ }^{3}$ Associate Professor, Lund University, Skane University Hospital, Department of Anaesthesiology and Intensive Care, S-22185, Lund, Sweden

\begin{abstract}
Background: Therapeutic fluids used in intensive care can have profound effects on coagulation, not only by dilution itself but also by other more complicated interactions. The aim of this in vitro study was to monitor the extent of dilutive coagulopathy induced by the most common therapeutic fluids and to attempt normalization of haemostasis by fibrinogen addition.
\end{abstract}

Methods: 8 patients were recruited from the intensive care unit. Native whole blood was drawn, diluted by $50 \%$ with 9 different fluids, and run through a Sonoclot Analyzer. This was then repeated with high dose fibrinogen (corresponding to an in vivo dose of $8 \mathrm{~g} / 70 \mathrm{~kg}$ ) added to the dilutions. The fluids used were Voluven, Venofundin, Volulyte, Tetraspan, Albumin 5\%, Macrodex, Gelofusine, Ringer's acetate and $\mathrm{NaCl}$. This covers the entire spectrum of fluids available in Sweden.

Results: A significant in vitro dilutive response compared to undiluted blood was seen for all synthetic colloid fluids but not for albumin or crystalloids. Dextran and Gelofusine's impact on coagulation parameters was greater than both $\mathrm{NaCl}$ and Ringer's acetate. The individual patient's response showed a high variability, which was reflected in high standard deviations. No significant improvement from fibrinogen addition could be seen on Sonoclot parameters.

Conclusion: The dilutive effects of resuscitation fluids at $50 \%$ dilution are more severe for synthetic colloids compared to alternative therapies. Fibrinogen addition did not affect the induced coagulopathy as measured by the Sonoclot.

\section{Introduction}

The Sonoclot, a less documented viscoelastic test than thrombelastography/thrombelastometry (TEG/ROTEM) has been used to monitor dilutive effects of different therapeutic fluids before. [1,2] Both hydroxyethyl starch (200/0.5), saline, Hartmann's solution, Gelofusine, Haemaccel, dextran 40, and albumin $4.5 \%$ have been evaluated. [3] No simultaneous comparison of all therapeutic fluids available: $\mathrm{NaCl}$, Ringer's acetate, $5 \%$ albumin, $6 \%$ dextran 70, starch 140/0.3, buffered starch 140/03 and gelatine has been performed.

Recently both in vitro and in vivo corrective treatments of dilutive coagulopathies with different coagulation factor concentrates, i.e. fibrinogen or a combination with fibrinogen + prothrombin complex concentrate or factor XIII have been successfully monitored with ROTEM/TEG. [4,5] A joint study of fibrinogen and recombinant factor VIIa treatment of dilutive coagulopathy has been performed on the Sonoclot, [6] but the field is lacking additional recent studies.

A new Sonoclot Coagulation and Platelet function analyser prototype has been developed (Figure 1). New additions include a glass bead test that has been designed to initiate coagulation in a more stable manner than previous tests. Also new are the Sonoclot parameter "Platelet Function" (PF) and the software SignatureViewer, where the clot contraction signal has improved. [7] Because it also factors in clot contraction, PF better reflects platelet function than the time to peak (TP) used in previous studies [8].

An important difference between the clotting of blood during in vivo hemostasis and the clotting of blood in vitro in a TEG/ROTEM/ Sonoclot is the lack of the vascular wall. An abnormality of platelet adhesion to the vessel wall can coexist with normal platelet aggregation. Both Sonoclot and TEG better evaluates platelet aggregation [7].

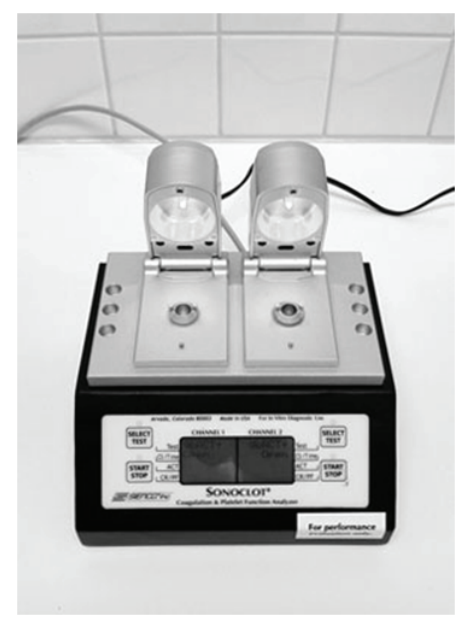

Figure 1: The Sonoclot.

*Corresponding author: Ulf Schött. MD, PhD, Associate Professor, Lund University, Skane University Hospital, Department of Anaesthesiology and Intensive Care, S-22185, Lund, Sweden. Tel: +46-46-171319; Fax +46-46-176050; E-mail: ulf.schott@skane.se

Received April 20, 2011; Accepted June 8, 2011; Published June 28, 2011

Citation: Lidgard E, Frigyesi A, Schött U (2011) Effects of High Dose Fibrinogen on in vitro Haemodilution with Different Therapeutic Fluids. J Blood Disord Transfus 2:106. doi:10.4172/2155-9864.1000106

Copyright: @ 2011 Lidgard E, et al. This is an open-access article distributed unde the terms of the Creative Commons Attribution License, which permits unrestricted use, distribution, and reproduction in any medium, provided the original author and source are credited. 
The purpose of this study was to use a Sonoclot Coagulation and Platelet function analyser to monitor in vitro effects of all available therapeutic fluids with blood from intensive care patients. This was done to preanalyse in vitro fluid effects before administering colloids to improve haemostatic safety in the intensive care.

\section{Materials and Methods}

After Regional Ethical Review Board Lund, Protocol DNR 2010/482, approval and informed consent (closest relative or patient after awakening), arterial blood was drawn from an indwelling radial artery catheter. A total of eight intensive care patients ( 2 female, 6 male) with no coagulation defects were studied. A continuous arterial catheter flush system was used and no blood was discarded before blood was sampled for the respective blood analyses (see below). The samples were collected in $5 \mathrm{ml}$ plastic syringes (BD Plastipak, Becton Dickinson, Spain).

Three Sonoclot Coagulation and Platelet function analyzers (Sienco Inc., Arvada, Colorado, US) with a total of 6 channels were used to study dilutive coagulation defects on native blood. Analyses were performed according to manufacturer's recommendations and Sonoclot parameters activated coagulation time (ACT), clot rate (CR) and platelet function $(\mathrm{PF})$ were digitally registered. All analyses were performed at 37 degrees Centigrade.

The Sonoclot measures the viscoelastic drag-impedance that fibrin and platelets in a whole blood sample impose upon the oscillating Sono-probe. A time-based graph reflects the different steps in the clotting whole blood sample. This graph is called the Sonoclot Signature (Figure 2), and the following parameters have defined normal values (in brackets below):

1. SonACT (Activated Coagulation Time) [100-155 s]: time taken for the first fibrin to form, and is defined as the time ( $\mathrm{min}$ ) it takes for the signature to move $1 \%$ upscale from the start of the graph (immersion response) - corresponds to aPTT and traditional ACT tests.

2. Clot Rate (CR) $[9-35 \% / \mathrm{min}]$ : the rate of increase in the clot impedance due to fibrin formation and

Polymerization (the slope of the Signature after SonACT) in \% of full scale per minute.

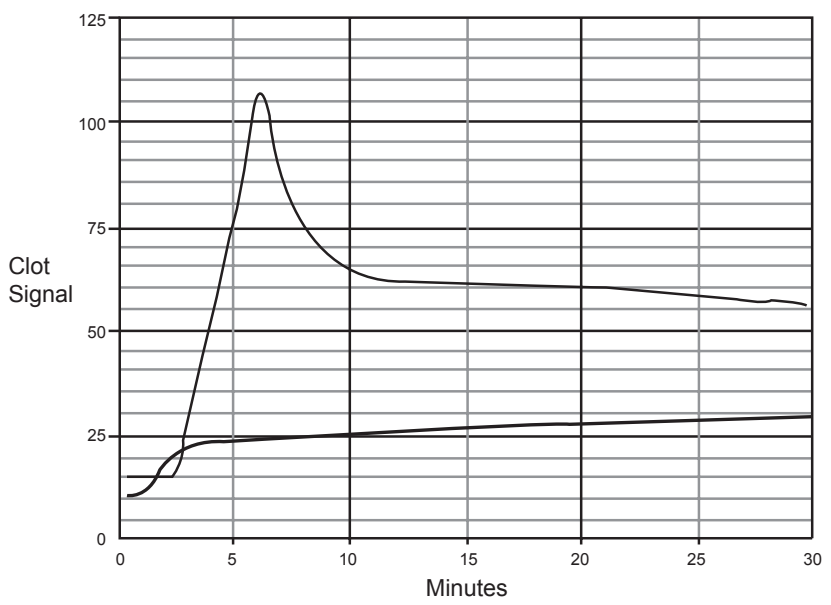

Figure 2: Two Sonoclot signatures from our work. Grey signature: undiluted blood sample. Black signature: 50\% Macrodex dilution sample.
3. Platelet Function $(\mathrm{PF})[>1,5]$ is the point at which the squeezing out of trapped serum in the contracting clot (sign of functioning platelets) exceeds the accumulation of clot bulk on the probe and can be described as the time to peak from the immersion response.

Undiluted blood was diluted to $50 \%$ with 9 different fluids: $\mathrm{NaCl}$ Ringer's acetate, 5\% albumin (CSL Behring), Macrodex (6\% dextran 70 (MEDA), Voluven (Kabi Fresenius), Venofundin (Braun), Volulyte (Kabi Fresenius), Tetraspan (MEDA), and Gelofusine (Braun). One $\mathrm{ml}$ blood and one $\mathrm{ml}$ of the respective colloid/crystalloid, was gently mixed in $5 \mathrm{ml}(75 \times 12 \mathrm{~mm})$ plastic tubes (Sarstedt, Numbrecht, Germany) and then pipetted into preheated Sonoclot cuvettes. Analyses began within 2 minutes from sampling.

Sonoclot analyses were performed with $0.36 \mathrm{ml}$ of undiluted native whole blood ; $0.36 \mathrm{ml}$ diluted (with the different fluids according to above) native blood.

The hemodilution effects on $\mathrm{pH}$, lactate and ionic calcium $\left(\mathrm{Ca}^{+}\right)$ level were tested with arterial blood gases analysed on an ABL 800 Flex (Radiometer, Copenhaguen, Denmark). The dilution effect was also controlled with whole blood APTT (activated partial thromboplastin time) and PT (prothrombin time) cuvettes on a HEMOCHRON Jr (ITC, Edison, US).

Then 50\% diluted native blood with the above fluids were in vitro spiked with fibrinogen concentrate (Haemocomplettan, CSL Behring, Marburg, Germany) corresponding to an in vivo dose of $8 \mathrm{~g} / 70 \mathrm{~kg}$. $(160 \mu \mathrm{l}$ of Haemocomplettan $20 \mathrm{mg} / \mathrm{ml}$ ) The effect of fibrinogen on undiluted and diluted native blood was then controlled with whole blood Sonoclot Coagulation and Platelet function analysers according to above.

\section{Statistical Analysis}

All data are shown as means ( \pm standard error of the mean, SEM). We employed Friedman's test, which is a non-parametric analogue to the repeated-measures ANOVA. Post-hoc analyses were carried out for all pairs of fluids and were considered significant if $\mathrm{p}<0.05$.

Differences in treatment with fibrinogen were evaluated with Wilcoxon's paired signed rank test and multiple testing was corrected for by the Bonferroni method, i.e. $<0.05$ divided by the number of tests was considered significant.

\section{Results}

The degree of haemodilution and effects on $\mathrm{pH}$ and ionic calcium level was tested with arterial blood gases analysed with an ABL 800 Flex. Median $\mathrm{pH}$ was $7.36 \mathrm{kPa}$, lactate $0.7 \mathrm{mmol} / \mathrm{L}$ and $\mathrm{Ca} 2^{+} 1.4 \mathrm{mmol} / \mathrm{L}$. Hemoglobin was halfed from 120-110 to 60-55 g/L.

The effects of the in vitro dilution on Hemochron Jr changed median PT from 1.2 to 2.1 INR (International Normalised Ratio) and APTT from 43.2 to 61 seconds (whole blood APTT has $>5$ seconds higher normal values than citrated plasma APTT). When fibrinogen was added to the diluted blood, whole blood APTT and PT were normalised.

Effects on dilution and corrective treatment with fibrinogen on the Sonoclot are shown in figure 3-5 for Activated clotting time (ACT), Clot Rate (CR) and Platelet function (PF) Sonoclot parameters. The dilution response compared to undiluted controls was significant for $\mathrm{CR}$ and $\mathrm{PF}$ but was negligible in ACT values. 
ACT

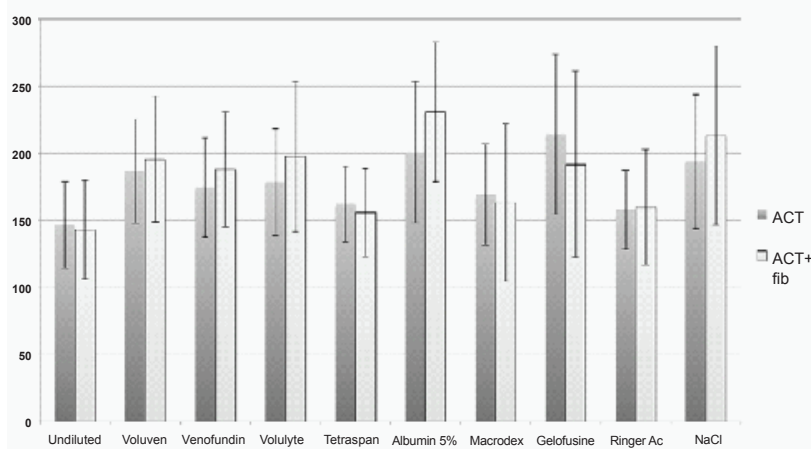

Figure 3: Activated Clotting Time values before and after fibrinogen addition.

CR

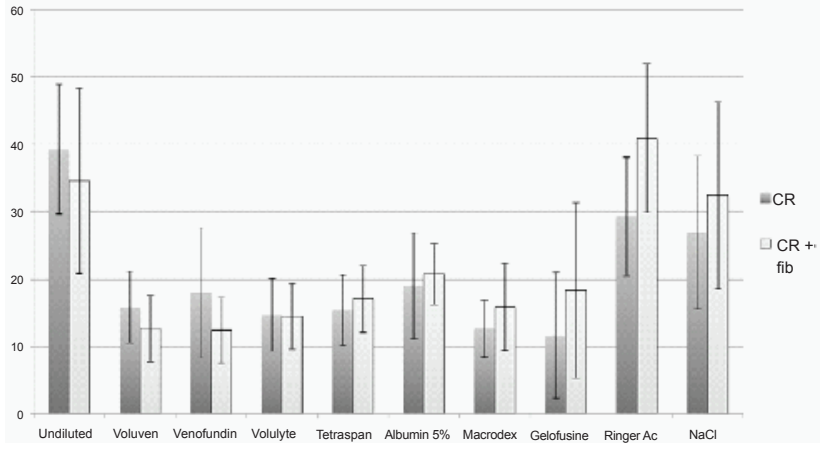

Figure 4: Clot rate values before and after fibrinogen addition.

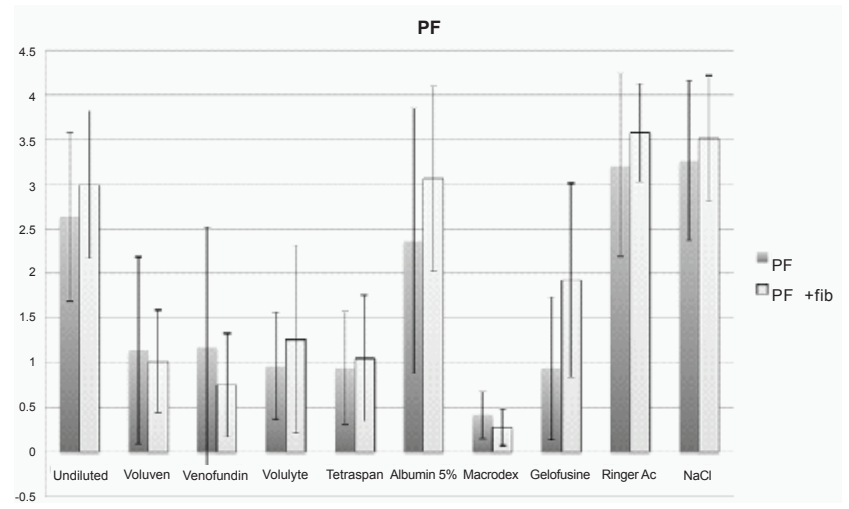

Figure 5: Platelet function values before and after fibrinogen addition.

No colloid-crystalloid distinction was found in the ACT parameter. Significant $(\mathrm{P}<0.05)$ dilution responses compared to undiluted controls were present for Voluven, Albumin, Gelofusine and $\mathrm{NaCl}$. Significant were also the differences between Gelofusine and Ringer's acetate, and between Gelofusine and Tetraspan.

Most diluted values (72\%) were outside the reference interval specified $(<155 \mathrm{~s})$ by the manufacturer. The addition of fibrinogen did not affect Activated Coagulation Time in a significant manner.

The clot rate pattern shows a significant drop in Voluven,
Venofundin, Volulyte, Tetraspan, Macrodex, and Gelofusine samples both with and without fibrinogen, the crystalloids faring better in comparison. No significant changes between undiluted blood and Albumin, Ringer's acetate, or $\mathrm{NaCl}$ were shown. Adding fibrinogen to diluted and undiluted samples did not have a significant impact on clot rate.

Gelofusine's negative influence on CR was significantly stronger compared to Ringer's acetate and $\mathrm{NaCl}$, as was the effect of Macrodex.

Our study showed a significant decrease in Platelet function (PF) for Macrodex. Other solutions failed to show a significant dilutive response, and no significant differences could be established between any other pair of fluids. Attempted correction with fibrinogen was not successful.

\section{Discussion}

\section{Colloid induced dilutive coagulopathy}

Together with hypothermia and acidosis, coagulopathy is one of the three conditions forming the bleeding patients so called trauma triad of death. Contributing factors to coagulation dysfunction include hypothermia and acidosis themselves, [9] emphasizing the interconnection between the members of the triad. [10] dilution is also a common culprit, the complexity of coagulation making it easy to disrupt. If given in large enough amounts, the fluids used trying to correct hypotension lead to coagulation disorders simply from the dilution of platelets and coagulation factors.

Simple dilution does not explain everything, however, as the haemodilution incurred when treating patients with therapeutic fluids tends to depress coagulation more or less profoundly depending on the fluid used. A larger decrease in coagulation markers than expected from the dilution itself has been observed for colloid diluted blood, with the impact corresponding in part to molecular size. High dalton-range HES solutions and dextran are generally seen as the worst offenders. [11] The mechanism proposed is interference with fibrin polymerization. [12] Synthetical colloid fluids in general seem to have a more negative impact compared to crystalloid solutions, [11,13] although results from some other studies appear to contradict these findings. [14] The confusion may stem from the many separate methodologies used in the research, amount of dilution, or the choice of analysis [3].

As findings remain inconclusive no individual treatment has become global standard and in Sweden the choice of fluid is largely up to the physician in charge. Many synthetic colloids, including starches and gelatine-based fluids, are in active use in the country. The choice of fluids in this study reflects the spectrum available clinically, ranging from different starch-based colloid solutions to crystalloids. Several starch colloids exist in buffered forms which are claimed to have less impact on coagulation, [15] which is why they were included in the study.

Correction of this evidently multifactorial process of coagulopathy can be equally as challenging as understanding the disorder in itself Lately use of fibrinogen as a treatment option has become widespread, following the discovery that fibrinogen plasma levels are the first of the procoagulatory proteins to drop to critical levels in bleeding patients. [16] Fibrinogen synthesis and activity is affected negatively by several of the same factors as coagulation in general like hypothermia and acidosis. [17] Although alternative therapies like fresh frozen plasma contain fibrinogen, the amounts are not high enough to compensate for the losses. [18] Use of high dose (up to 8g) fibrinogen 
like Haemocomplettan has in studies been proven to reconstitute or improve coagulation markers after its administration, both in vitro and in vivo. [19,20] Fibrinogen has also shown promise in correcting dilutive coagulopathy regardless of the fluid used, which hasn't been seen for all similar therapies, most notably perhaps activated factor VII $[13,20]$.

\section{Coagulation monitoring}

Getting an accurate and meaningful measurement of a patient's coagulative state is not straightforward. Viscoelastic test such as thromboelastography and thromboelastometry in the form of TEG and ROTEM have become widespread methods of measuring coagulation and their usefulness in the point of care setting has been shown. [21] They remain however largely in the realm of research, as the methodology surrounding the tests hasn't been sufficiently standardized. Recent efforts to prove a low inter-laboratory variance have failed, marking the need for more research before full clinical use can be realistic. [22] None the less, later studies are calling for a more active use of viscoelastic tests like ROTEM, seeing potential in perioperative coagulation monitoring en lieu of standard laborative tests like activated partial thromboplastin time (aPTT) or protrombin time (PT) [23].

The Sonoclot Coagulation \& Platelet Function Analyzer, while also a viscoelastic test, does not have as much documentation. Still, evidence of it's worth is mounting, [24] and some suggest that complementary use of TEG and Sonoclot is viable. In effect, parallel use of the devices has already been applied in a research setting [6].

The Sonoclot analyses coagulation in a broader sense, but especially with the new glass bead test used in this study, the focus is on platelet function. Considering that many fibrinogen studies $[19,20]$ have been performed on the ROTEM or TEG with the platelet function incapacitated with cytochalasin (known as fib-tem and modified TEG) this could possibly have contributed to the lack of fibrinogen response in our samples. Still, recent findings suggest a positive fibrinogen response also in platelet function so this cannot fully explain the lack of reaction [25].

Sonoclot uses native blood for measurement, as opposed to the citrate blood most often used in TEG and ROTEM. Slightly impractical as this may be, citrated blood has been implied to interfere with thrombin generation and thus produce errors in coagulation assessment, making native blood a more attractive choice in this regard $[26,27]$.

\section{Conclusions}

Our perception of our study's strong points includes the use of whole blood, as well as the documentation of the full range of available fluids. We also feel using actual ICU patients instead of healthy volunteers better reflects the true nature of coagulation disorders. Of course, these points can just as well be turned against us. The ICU patients suffered from several disparate diseases making the risk of interference with results tangible. The use of whole blood is cumbersome for most laboratories and may lower the relevancy of our findings. Other weak aspects of our study are our small test population, worsened by the inherent variability of the Sonoclot machine [28].

Our results show that a haemodilution with therapeutic fluids to $50 \%$ does indeed affect most Sonoclot parameters. They also tell us that this effect is far from uniform over the wide range of fluids used. This was expected. A split between synthetic colloid and crystalloid fluids was also seen, significant for the CR parameter and perhaps existing as a trend in PF. We believe this trend would have become significant if our study had a higher power. Albumin stands out as the only colloid that could not be shown to significantly affect coagulation by our dilution.

Dextran had a significantly higher impact on platelet function compared to other fluids; a finding which is in line with previous research [29]. Our data suggests using dextran cautiously when platelet function is critical.

Our hypothesis of fibrinogen correcting or at least ameliorating the dilutive coagulopathy could not be proven correct; no significant changes could be accomplished in any of the three parameters. Previous research by other groups cited above have shown positive results of fibrinogen addition in vitro but only one of these used the Sonoclot and did not use the same methodology surrounding the tests as we have. Our direct ICU-patient approach and use of native blood may account for our inability to reproduce these results.

In summary, our work reinforces the notion that synthetic colloid fluids are more detrimental to coagulation than albumin and crystalloid alternatives. Attempted fibrinogen correction was in spite of previous promising work not successful in our setting.

\section{Acknowledgements}

Eric Lidgard and Attila Frigyesi have no conflicts of interest. Ulf Schött has recieved speaker fees 2009 and 2010 from CSL Behring, who also provided the Haemocomplettan used in this in vitro study. All laboratory costs were covered by grants from Skåne University Hospital in Lund, Sweden.

\section{References}

1. Brazil EV, Coats TJ (2000) Sonoclot coagulation analysis of in-vitro haemodilution with resuscitation solutions. J R Soc Med 93: 507-510.

2. Konrad CJ, MarkI TJ, Schuepfer GK, Schmeck J, Gerber HR, et al. (2000) In vitro effects of different medium molecular hydroxyethyl starch solutions and lactated Ringer's solution on coagulation using SONOCLOT. Anesth Analg 90: 274-279.

3. Coats T J, Brazil E, Heron M (2006) The effects of commonly used resuscitation fluids on whole blood coagulation. Emerg Med J 23: 546-549.

4. Fries D, Haas T, Klingler A, Streif W, Klima G, et al. (2006) Efficacy of fibrinogen and prothrombin complex concentrate used to reverse dilutional coagulopathy-a porcine model. Br J Anaesth 97: 460-467.

5. Haas T, Fries D, Velik-Salchner C, Reif C, Klingler A, et al. (2008) The in vitro effects of fibrinogen concentrate, factor XIII and fresh frozen plasma on impaired clot formation after 60\% dilution. Anesth Analg 106: 1360-1365.

6. Ganter MT, Schmuck S, Hamiel CR, Wischmeyer PE, Heule D, et al. (2008) Monitoring Recombinant Factor VIla Treatment: Efficacy Depends on High Levels of Fibrinogen in a Model of Severe Dilutional Coagulopathy. Journal of Cardiothoracic and Vascular Anesthesia 22: 675-680.

7. Berezina TL, Zaets SB, Morgan C, Spillert CR, Kamiyama M, et al. (2002) Influence of storage on red blood cell rheological properties. Journal of surgical research 102: 6-12.

8. Schott U, Nilsson LG, Broman M, Engstrom M (2010) Monitoring of low molecular weight heparin anticoagulation during haemodialysis with a Sonoclo Analyzer. Perfusion 25: 191-196.

9. Engstrom M, Schott U, Romner B, Reinstrup P (2006) Acidosis impairs the coagulation: A thromboelastographic study. J Trauma 61: 624-628.

10. Johansson PI, Ostrowski SR, Secher NH (2010) Management of major blood loss: an update. Acta Anaesthesiol Scand 54: 1039-1049.

11. Van der Linden P, Ickx BE (2006) The effects of colloid solutions on hemostasis. Can J Anaesth 53: 530-539. 
Citation: Lidgard E, Frigyesi A, Schött U (2011) Effects of High Dose Fibrinogen on in vitro Haemodilution with Different Therapeutic Fluids. J Blood Disord Transfus 2:106. doi:10.4172/2155-9864.1000106

Page 5 of 5

12. De Lorenzo C, Calatzis A, Welsch U, Heindl B (2006) Fibrinogen concentrate reverses dilutional coagulopathy induced in vitro by saline but not by hydroxyethyl starch 6\%. Anesth Analg 102: 1194-1200.

13. Fenger-Eriksen C, Anker-Moller E, Heslop J, Ingerslev J, Sorensen B (2005) Thrombelastographic whole blood clot formation after ex vivo addition of plasma substitutes: improvements of the induced coagulopathy with fibrinogen concentrate. Br J Anaesth 94: 324-329.

14. Karoutsos S, Nathan N, Lahrimi A, Grouille D, Feiss P, et al. (1999) Thrombelastogram reveals hypercoagulability after administration of gelatin solution. Br J Anaesth 82: 175-177.

15. Boldt J, Mengistu A, Seyfert UT, Vogt A, Hellstern P (2007) The impact of medium molecular weight, low molar substitution hydroxyethyl starch dissolved in a physiologically balanced electrolyte solution on blood coagulation and platelet function in vitro. Vox Sang 93: 139-144.

16. Hiippala ST, Myllyla GJ, Vahtera EM (1995) Hemostatic factors and replacemen of major blood loss with plasma-poor red cell concentrates. Anesth Analg 81 360-365.

17. Fries D, Martini WZ (2010) Role of fibrinogen in trauma-induced coagulopathy $\mathrm{Br}$ J Anaesth 105: 116-121.

18. Chowdhury P, Saayman AG, Paulus U, Findlay GP, Collins PW (2004) Efficacy of standard dose and $30 \mathrm{ml} / \mathrm{kg}$ fresh frozen plasma in correcting laboratory parameters of haemostasis in critically ill patients. Br J Haematol 125: 69-73.

19. Solomon C, Pichlmaier U, Schoechl H, Hagl C, Raymondos K, et al. (2010) Recovery of fibrinogen after administration of fibrinogen concentrate to patients with severe bleeding after cardiopulmonary bypass surgery. Br J Anaesth 104 : 555-562.

20. Fries D, Innerhofer P, Reif C, Streif W, Klingler A, et al. (2006) The effect of fibrinogen substitution on reversal of dilutional coagulopathy: an in vitro model. Anesth Analg 102: 347-351.

21. Kaufmann CR, Dwyer KM, Crews J D, Dols SJ, Trask AL (1997) Usefulness of thrombelastography in assessment of trauma patient coagulation. J Trauma 42: $716-720$

22. Chitlur M, Sorensen B, Rivard GE, Young G, Ingerslev J, et al. (2011) J Standardization of thromboelastography: a report from the TEG-ROTEM working group. Haemophilia

23. Kozek-Langenecker SA (2010) Perioperative coagulation monitoring. Best Pract Res Clin Anaesthesiol 24: 27-40.

24. Yamada T, Katori N, Tanaka KA, Takeda J (2007) Impact of Sonoclo hemostasis analysis after Cardiopulmonary bypass on postoperative hemorrhage in cardiac surgery. J Anesth 21: 148-152.

25. Lang T, Johanning K, Metzler H, Piepenbrock S, Solomon C, et al. (2009) The effects of fibrinogen levels on thromboelastometric variables in the presence of thrombocytopenia. Anesth Analg 108: 751-758.

26. Fenger-Eriksen C, Ingerslev J, Tonnesen E, Sorensen B (2009) Citrate artificially masks the haemostatic effect of recombinant factor VIla in dilutional coagulopathy. Ann Hematol 88: 255-260.

27. White H, Zollinger C, Jones M, Bird R (2010) Can Thromboelastography performed on kaolin-activated citrated samples from critically ill patients provide stable and consistent parameters? Int J Lab Hematol 32: 167-173.

28. Ekback G, Carlsson O, Schott U (1999) Sonoclot coagulation analysis: a study of test variability. J Cardiothorac Vasc Anesth 13: 393-397.

29. Kozek-Langenecker S A (2009) Influence of fluid therapy on the haemostatic system of intensive care patients. Best Pract Res Clin Anaesthesiol 23: 225-236. 